\title{
A PESCA DA ALBACORA-LAGE (Thunnus albacares) REALIZADA PELA FROTA BRASILEIRA DE VARA E ISCA- VIVA NO SUDOESTE DO OCEANO ATLÂNTICO
}

\author{
TEIXEIRA DOS SANTOS, J.A. ${ }^{1,2} \&$ H.A. ANDRADE ${ }^{1}$ \\ 1 UNIVALI - CTTMar, Rua Uruguai, 458. CEP 88302-202, Itajaí-SC, Brasil; \\ 2 PPGOB - FURG/Depto. Oceanografia, Av. Itália km8 s/n. CEP 96201-900, Rio Grande- \\ RS, Brasil. \\ joao.oceano@gmail.com
}

RESUMO

\begin{abstract}
No sudoeste do Oceano Atlântico, as capturas de albacora-lage (Thunnus albacares) são relativamente altas, mas o bonito-listrado (Katsuwonus pelamis) é a espécie alvo. Entretanto, devido ao maior valor de mercado, os pescadores têm se interessado em tentar aumentar as capturas e os rendimentos da albacora-lage no sudeste e sul do Brasil. Com o objetivo de reunir informações para melhorar a pescaria da albacora-lage, foram analisadas as variações espaçotemporais dos padrões de pesca (esforço, captura e CPUE) na costa do Brasil. Os resultados preliminares sugerem que a vulnerabilidade da albacora-lage é grande ao longo de toda a área de pesca tradicional da frota de vara e isca-viva no Brasil. No entanto, os rendimentos são especialmente altos no segundo trimestre e no sul (ao sul de $30^{\circ} \mathrm{S}$ ). Alem disso, dispositivos agregadores (e.g. plataformas de petróleo e bóias ancoradas) provaram aumentar os rendimentos da albacoralage.
\end{abstract}

Palavras-chave: Albacora-lage, Thunnus albacares, Isca-viva, Captura, CPUE, Variações sazonais, Objetos flutuantes.

\section{THE YELLOWFIN TUNA (Thunnus albacares) FISHERY CARRIED OUT BY THE BRAZILIAN BAITBOAT FLEET IN THE SOUTHWEST ATLANTIC OCEAN}

\begin{abstract}
In the southwest Atlantic Ocean, the catches of yellowfin tuna (Thunnus albacares) are relatively high, but skipjack tuna (Katsuwonus pelamis) is the target species. However, due to the high market value, fishermen have been interested in trying to increase the yellowfin catches and yields in the southeast and south Brazil. In order to gather information to improve yellowfin fishery we analyzed the spatio-temporal variations of the fishing patterns (effort, catch and CPUE) off Brazil. The preliminary results obtained suggest that the vulnerability of yellowfin tuna is large all over the traditional baitboat fishing ground off Brazil. However, the profits are especially high in the second quarter and in south (southward of $30^{\circ} \mathrm{S}$ ). Furthermore, aggregating devices (e.g. oilrig platforms and drifting or anchored buoys) proved to enhance yellowfin tuna yields.
\end{abstract}

Keywords: Yellowfin tuna, Thunnus albacares, Live bait, Catch, Effort, CPUE, Seasonal variations, Floating objects.

\section{INTRODUÇÃO}

A albacora-lage (Thunnus albacares) é uma das principais espécies explotadas na costa brasileira, correspondendo a uma porção significativa das capturas das frotas de vara e isca-viva e espinhel de superfície. Em 2000 e 2001, por exemplo, sua captura anual foi superior a 6.100 toneladas (ICCAT, 2002), o que representa cerca de $14 \%$ da captura total de 
atuns no Brasil. Em desembarques realizados em Santa Catarina, a albacora-lage representa cerca de $7,5 \%$ das capturas da frota de vara e isca-viva (Santos \& Andrade, 2003) e aproximadamente $13 \%$ do total capturado pela frota de espinhel de superfície (UNIVALI/CTTMar, 2003a). Em ambos os casos, a albacora-lage é a segunda espécie mais capturada, ficando atrás apenas do bonito-listrado (Katsuwonus pelamis) na pesca de vara e isca-viva e do espadarte (Xiphias gladius) na pesca de espinhel de superfície.

Os desembarques de albacora-lage da frota de vara e isca-viva em Santa Catarina são muito superiores às da frota de espinhel de superfície. Em 2002, das 927 t de albacoralage desembarcadas no estado (UNIVALI/ CTTMar, 2003b), cerca de $731 \mathrm{t}$ (cerca de 80\%) vieram de desembarques da frota de vara e iscaviva nos portos pesqueiros do Itajaí e Navegantes. A área de pesca dessa frota se estende de $20^{\circ} \mathrm{S}$ a $35^{\circ} \mathrm{S}$ no sudoeste do Atlântico, indo da costa até $37^{\circ} \mathrm{W}$ (Vilela, 1990; Vilela \& Castelo, 1991; Andrade, 2003) (Figura 1). Em geral, as embarcações se concentram ao longo da borda da plataforma continental, onde as variáveis ambientais (e.g. temperatura, frentes oceanográficas) são favoráveis à captura do bonito-listrado, que é o alvo da pescaria (Andrade, 2003). No entanto, uma vez que o valor de mercado de exemplares de albacoralage maiores que $6 \mathrm{~kg}$ é $25 \%$ superior ao valor do bonito-listrado, os mestres de pesca têm manifestado interesse em aumentar as captura de albacora-lage há alguns anos. Portanto, conhecimentos a respeito do potencial pesqueiro da albacora-lage no sudoeste do oceano Atlântico são importantes para gerar subsídios para novas estratégias de explotação deste recurso. A importância de tais conhecimentos sobre a pescaria cresce ainda mais devido à inexistência de uma cota de captura de albacora-lage regulamentada pela International Commission for the Conservation of Atlantic Tunas (ICCAT) para o sudoeste do Atlântico. Dessa forma, o objetivo deste trabalho, que consiste em identificar cenários (áreas e trimestres) favoráveis à pesca da albacora-lage na costa Brasileira, vem de encontro à necessidade de aquisição de novas informações. Para atingir esse fim, foram utilizados dados de pesca coletados nos portos de Itajaí e Navegantes na análise das variações espaço-temporais de variáveis (e.g. captura e esforço) relacionadas à pesca da albacora-lage realizada pela frota de vara e isca-viva.

\section{MATERIAIS E MÉTODOS}

As amostragens foram realizadas nos portos pesqueiros de Itajaí e Navegantes em Santa Catarina, onde atualmente ocorre a maior parte dos desembarques da frota de vara $e$ isca-viva no Brasil. As capturas anuais do bonito-listrado (alvo da pescaria) atingem nestes portos mais de $65 \%$ da captura total brasileira (Paiva, 1997; Meneses de Lima et al., 2000). As embarcações que desembarcam em Itajaí e Navegantes pescam em toda a área de pesca tradicional da frota de vara e isca-viva, de $20^{\circ} \mathrm{S}$ a $35^{\circ} \mathrm{S}$ (Figura 1). Assim, os desembarques realizados em Santa Catarina são representativos das capturas obtidas na margem sudoeste do Oceano Atlântico.

Este trabalho contém os resultados de análises de desembarques da frota de vara e isca-viva referentes aos anos de 2001 e 2002. Três fontes de informação sobre os desembarques (entrevistas, mapas de bordo e fichas de produção) foram usadas. Detalhes sobre o funcionamento e validade dessas fontes de dados nas pescarias de atuns podem ser encontrados em Santos \& Andrade (2003). Estes autores indicaram que alguns mecanismos de comparação entre essas fontes de informação resultam em estimativas relativamente acuradas. Neste trabalho foram usados os mesmos procedimentos descritos em Santos \& Andrade (2003).

A análise das variações espaço-temporais dos padrões de pesca da albacora-lage em 
2001 foi baseada em 172 entrevistas, 123 mapas de bordo e 111 fichas de produção, totalizando 274 desembarques e 3.423 dias de mar (Tabela 1). As análises das capturas de 2002 foram baseadas em 151 entrevistas, 117 mapas de bordo e 163 fichas de produção, resultando em 260 desembarques e 2.952 dias de mar. Os dados de pesca utilizados neste trabalho referem-se a 33 barcos nacionais de vara e isca-viva, dos quais 28 são de Santa Catarina, 3 do Rio de janeiro e 2 do Rio Grande do Sul.

Os dados de captura são apresentados em toneladas e o esforço em "dias efetivos de pesca" (dias em que houve pelo menos uma tentativa de captura com liberação de isca).

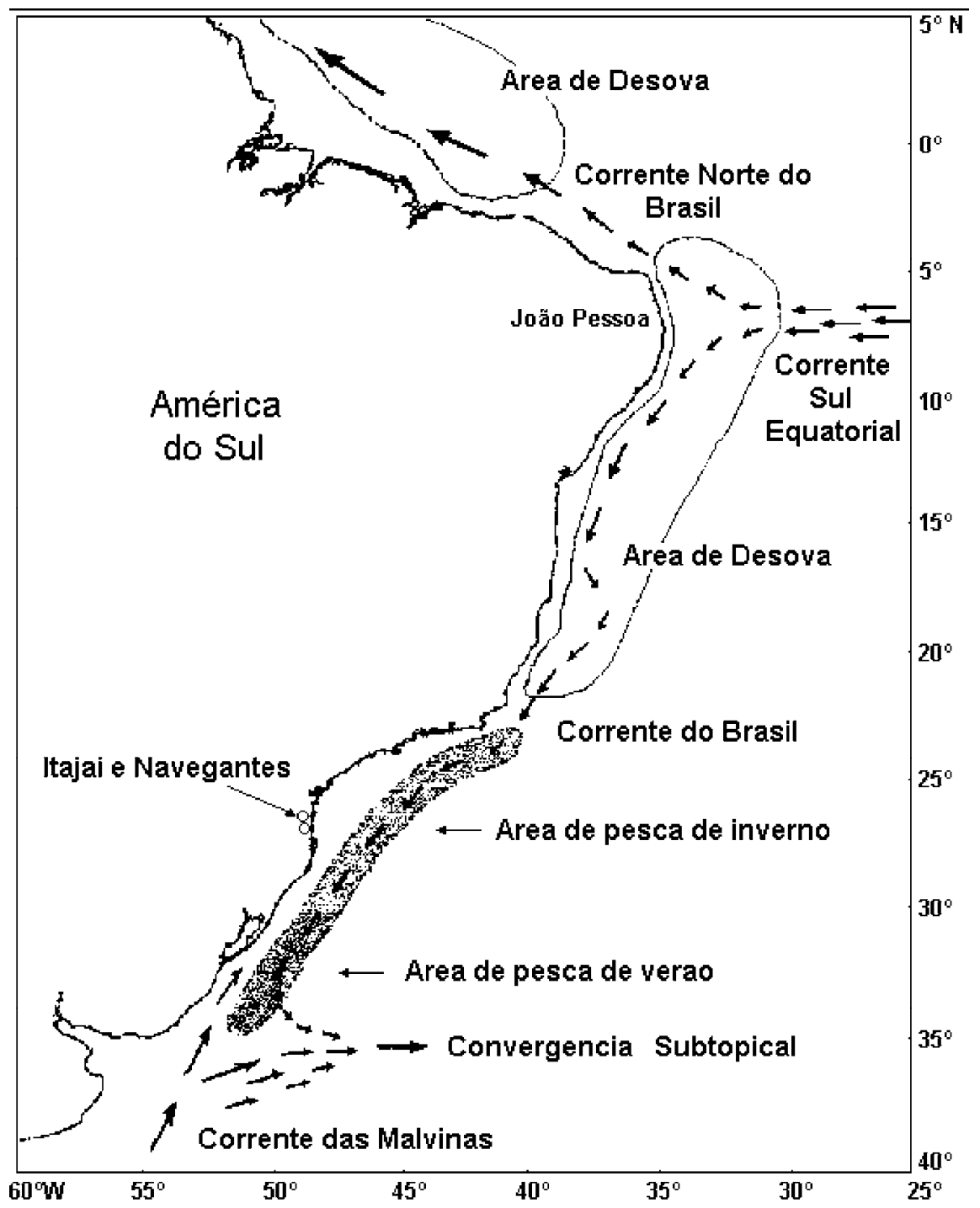

Figura 1 - Área de pesca e de reprodução do bonito listrado (Katsuwonus pelamis), para o litoral brasileiro (retirada de Matsuura e Andrade, 2000). 
Para plotar os resultados nos mapas, os dados foram agrupados por trimestre e quadrante $\left(1^{\circ} \times 1^{\circ}\right)$ seguindo o procedimento tradicionalmente utilizado pela ICCAT. A captura por unidade de esforço (CPUE) é apresentada na forma de médias ponderadas pelo esforço de pesca para cada quadrante e trimestre. Portanto, a CPUE $(U)$ é dada por:

$$
U i=\frac{\sum C i}{\sum f i}
$$

onde $C$ e $f$ são a captura e o esforço da frota nos i eventos de pesca para esse cenário (i.e. quadrante e trimestre). Este procedimento segue a metodologia tradicional exposta em Quinn et al. (1982).

\section{RESULTADOS}

Dados de pesca de 2001

No primeiro trimestre de 2001, a frota de vara e isca-viva apresentou uma ampla distribuição do esforço de pesca, atuando de $24^{\circ} \mathrm{S}$ a $34^{\circ} \mathrm{S}$ no sudoeste do Atlântico (Figura 2A). No restante do ano, as operações de pesca

Tabela 1 - Resumo dos dados frota brasileira de vara e isca-viva utilizados na análise das variações espaçotemporais dos padrões de pesca da albacora-lage (Thunnus albacares) em 2001 e 2002.

\begin{tabular}{cccc}
\hline Ano & Trimestre & $\begin{array}{c}\text { Número de } \\
\text { desembarques }\end{array}$ & Dias no mar \\
\hline 2001 & 1 & 54 & 875 \\
& 2 & 67 & 1116 \\
& 3 & 46 & 913 \\
& 4 & 45 & 519 \\
2002 & 1 & 57 & 635 \\
& 2 & 78 & 910 \\
& 3 & 53 & 934 \\
& 4 & 26 & 473 \\
\hline
\end{tabular}

concentraram-se principalmente entre $24^{\circ} \mathrm{S} \mathrm{e}$ $28^{\circ} \mathrm{S}$ (Figuras 2B, 2C e 2D). Do verão para o inverno a frota deslocou-se gradualmente para áreas de pesca mais ao norte. Do inverno para o verão houve uma inversão deste processo, e boa parte das embarcações de vara e isca-viva passaram a utilizar novamente as áreas de pesca situadas ao sul de $30^{\circ} \mathrm{S}$.

No primeiro trimestre de 2001, as maiores capturas de albacora-lage foram obtidas no sul, principalmente entre as latitudes de $31^{\circ} \mathrm{S}$ e $32^{\circ} \mathrm{S}$ e as longitudes de $49^{\circ} \mathrm{W}$ e $50^{\circ} \mathrm{W}$ (Figura $3 \mathrm{~A})$. A captura total obtida nesta área representou cerca de $26 \%$ das capturas de albacoralage amostradas no primeiro trimestre (72 t). No segundo trimestre, a maior captura foi obtida entre as latitudes de $27^{\circ} \mathrm{S}$ e $28^{\circ} \mathrm{S}$ e as longitudes de $47^{\circ} \mathrm{W}$ e $48^{\circ} \mathrm{W}$ (Figura 3B). O montante capturado nesta área correspondeu à cerca de $40 \%$ da captura total de albacora-lage amostrada no segundo trimestre (576,6 t). De acordo com informações de entrevistas e mapas de bordo, $163 \mathrm{t}$ (cerca de $72 \%$ ) das $230 \mathrm{t}$ capturadas neste quadrante vieram de lances de pesca realizados nas proximidades de objetos flutuantes (fixos ou a deriva) e de plataformas de extração de petróleo. No terceiro e no quarto trimestres, o quadrante $25^{\circ} \mathrm{S}-26^{\circ} \mathrm{S} /$ $45^{\circ} \mathrm{W}-46^{\circ} \mathrm{W}$ apresentou as maiores capturas totais de albacora-lage (Figuras 3C e 3D). Das 48 t obtidas neste quadrante durante o terceiro trimestre, cerca de $85 \%$ foram obtidas em lances de pesca efetuados nas imediações de uma plataforma de extração de petróleo. No quarto trimestre, cerca de $97 \%$ da albacoralage capturada nesse quadrante foi obtida no mesmo local.

De maneira geral, as maiores CPUEs da albacora-lage em 2001 ocorreram no segundo trimestre (Figura 4B). A área sul (ao sul de $30^{\circ} \mathrm{S}$ ) sempre foi mais rentável quando foi utilizada pela frota de vara e isca-viva (Figuras 4A, $4 \mathrm{~B}$ e 4D). Já a área norte (ao norte de $27^{\circ} \mathrm{S}$ ) tendeu a apresentar CPUEs mais baixas, principalmente no primeiro e no quarto trimestres (Figuras 4A e 4D). No segundo e no terceiro 
trimestres, as CPUEs nessa área foram sensivelmente maiores, principalmente ao largo do estado do Rio de Janeiro (Figuras 4B e 4C).

Dados de pesca de 2002

O padrão de distribuição do esforço de pesca da frota de vara e isca-viva em 2002 foi bastante similar ao apresentado em 2001 (Figura 5). As principais diferenças ocorreram no primeiro e no segundo trimestre. No primeiro trimestre de 2002 (Figura 5A), houve proporci- onalmente uma menor concentração do esforço em áreas de pesca ao sul de $30^{\circ} \mathrm{S}$ (cerca de $17 \%$ do total em 2002 contra aproximadamente $40 \%$ em 2001). Já no segundo trimestre (Figura 5B), houve uma maior distribuição das operações de pesca ao longo das longitudes, indicando que a frota pescou em áreas mais rasas e mais profundas do que em 2001 (Figura $2 \mathrm{~B}$ ).

Assim como em 2001, grande parte das capturas de albacora-lage veio de lances de pesca realizados nas imediações de objetos
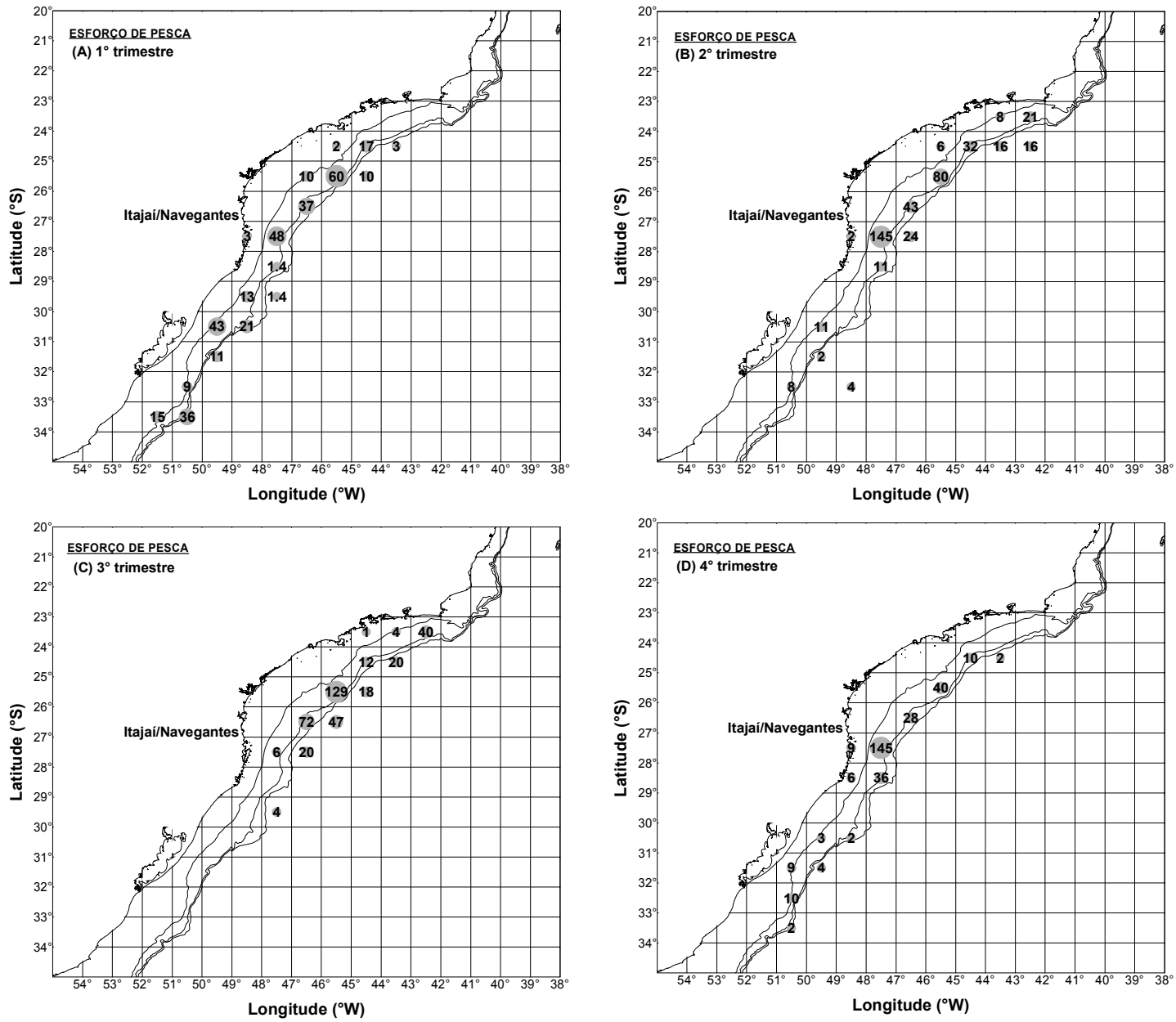

Figura 2 - Distribuição espaço-temporal do esforço de pesca da frota de vara e isca-viva (dias efetivos de pesca) em 2001: (A) $1^{\circ}$ trimestre, (B) $2^{\circ}$ trimestre, (C) $3^{\circ}$ trimestre e (D) $4^{\circ}$ trimestre. As linhas contínuas paralelas à linha de costa representam as isóbatas de 100, 200 e 500 metros, respectivamente. 
flutuantes e de plataformas de extração de petróleo. A principal diferença ficou por conta do quadrante $25^{\circ} \mathrm{S}-26^{\circ} \mathrm{S} / 45^{\circ} \mathrm{W}-46^{\circ} \mathrm{W}$ no primeiro trimestre (Figura 6A), onde, apesar de terem sido capturadas $18 \mathrm{t}$ de albacora-lage, não foram reportados lances de pesca nas proximidades de uma plataforma de petróleo que é tradicionalmente utilizada pelas embarcações de vara e isca-viva neste quadrante.

O padrão de variação espaço-temporal da CPUE da albacora-laje em 2002 (Figura 7) foi muito semelhante ao observado em 2001 (Figura 4), com o maior rendimento médio sendo obtido novamente no segundo trimestre e no quadrante $31^{\circ} \mathrm{S}-32^{\circ} \mathrm{S} / 49^{\circ} \mathrm{W}-50^{\circ} \mathrm{W}$ (Figura 7B), onde foram capturadas cerca de $16 \mathrm{t}$ por dia efetivo de pesca junto a uma bóia fixa. A principal diferença entre os dois anos foi o número expressivo de lances nulos que resultaram em CPUEs médias iguais a zero, basicamente na plataforma externa, entre $23^{\circ} \mathrm{S} \mathrm{e}$ $27^{\circ} \mathrm{S}$ (Figura 7B). Além disso, houve em 2002
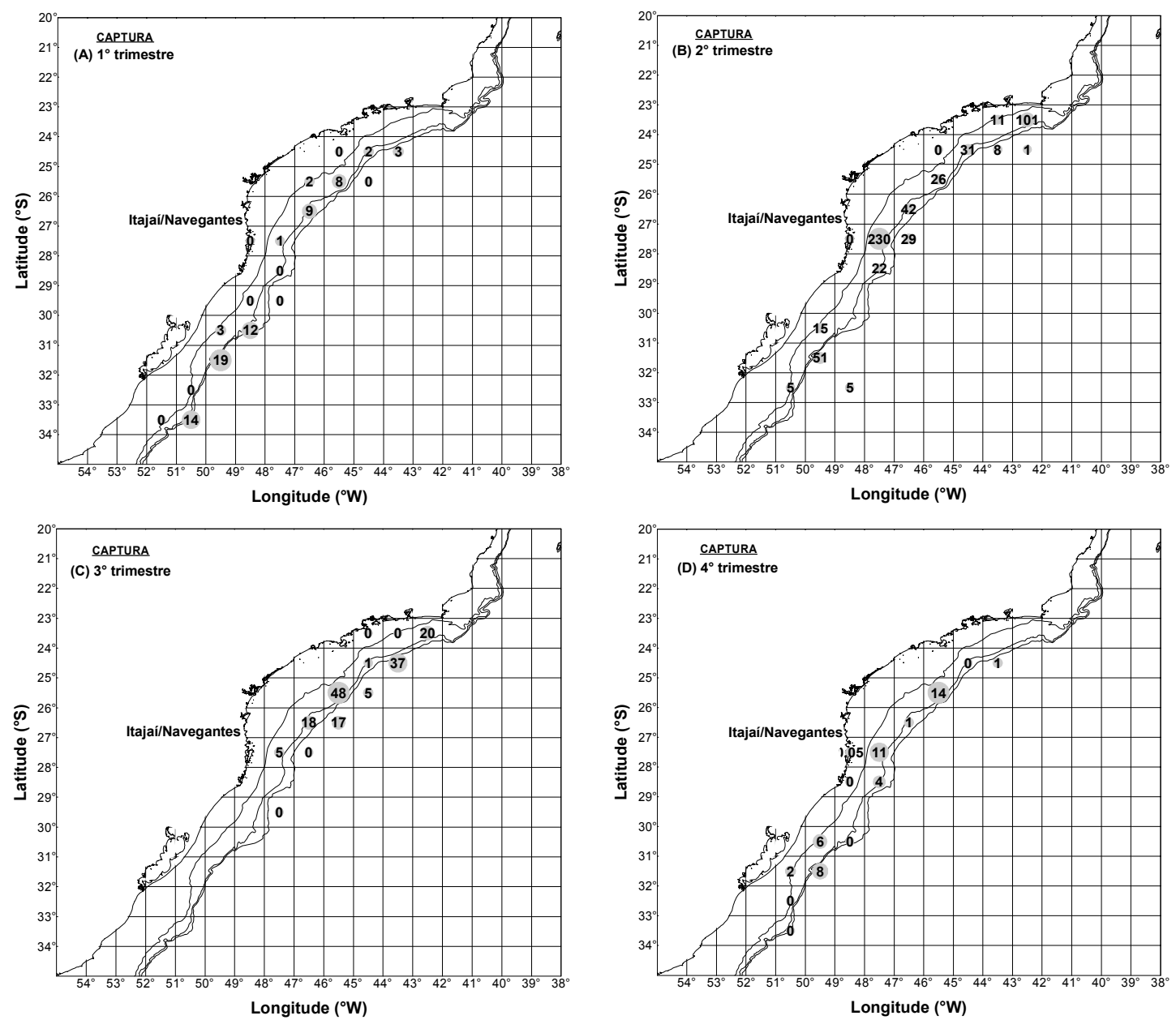

Figura 3 - Distribuição espaço-temporal das capturas de albacora-lage (toneladas) obtidas pela frota de vara e iscaviva em 2001: (A) $1^{\circ}$ trimestre, (B) $2^{\circ}$ trimestre, (C) $3^{\circ}$ trimestre e (D) $4^{\circ}$ trimestre. As linhas contínuas paralelas à linha de costa representam as isóbatas de 100, 200 e 500 metros, respectivamente. 
um grande incremento na CPUE média do quadrante $25^{\circ} \mathrm{S}-26^{\circ} \mathrm{S} / 44^{\circ} \mathrm{W}-45^{\circ} \mathrm{W}$ (Figura $7 \mathrm{C}$ ), onde, de acordo com as entrevistas e mapas de bordo analisados, houve eventos em que foram capturadas até $8 \mathrm{t}$ por dia de pesca nas proximidades de uma bóia fixa.

\section{DISCUSSÃO}

Os resultados obtidos a partir da análise das variações espaço-temporais dos pa- drões de pesca indicaram que as plataformas de extração de petróleo, bóias ancoradas pelos pescadores e objetos flutuantes a deriva desempenham um importante papel na pesca da albacora-lage realizada pela frota de vara e isca-viva no Brasil. Tanto a frota nacional como a arrendada geralmente pescam nas proximidades de objetos flutuantes naturais e artificiais e de plataformas de petróleo (UNIVALI/ CTTMar, 2003a; Andrade, 2003). A influência dos objetos flutuantes foi bastante expressiva
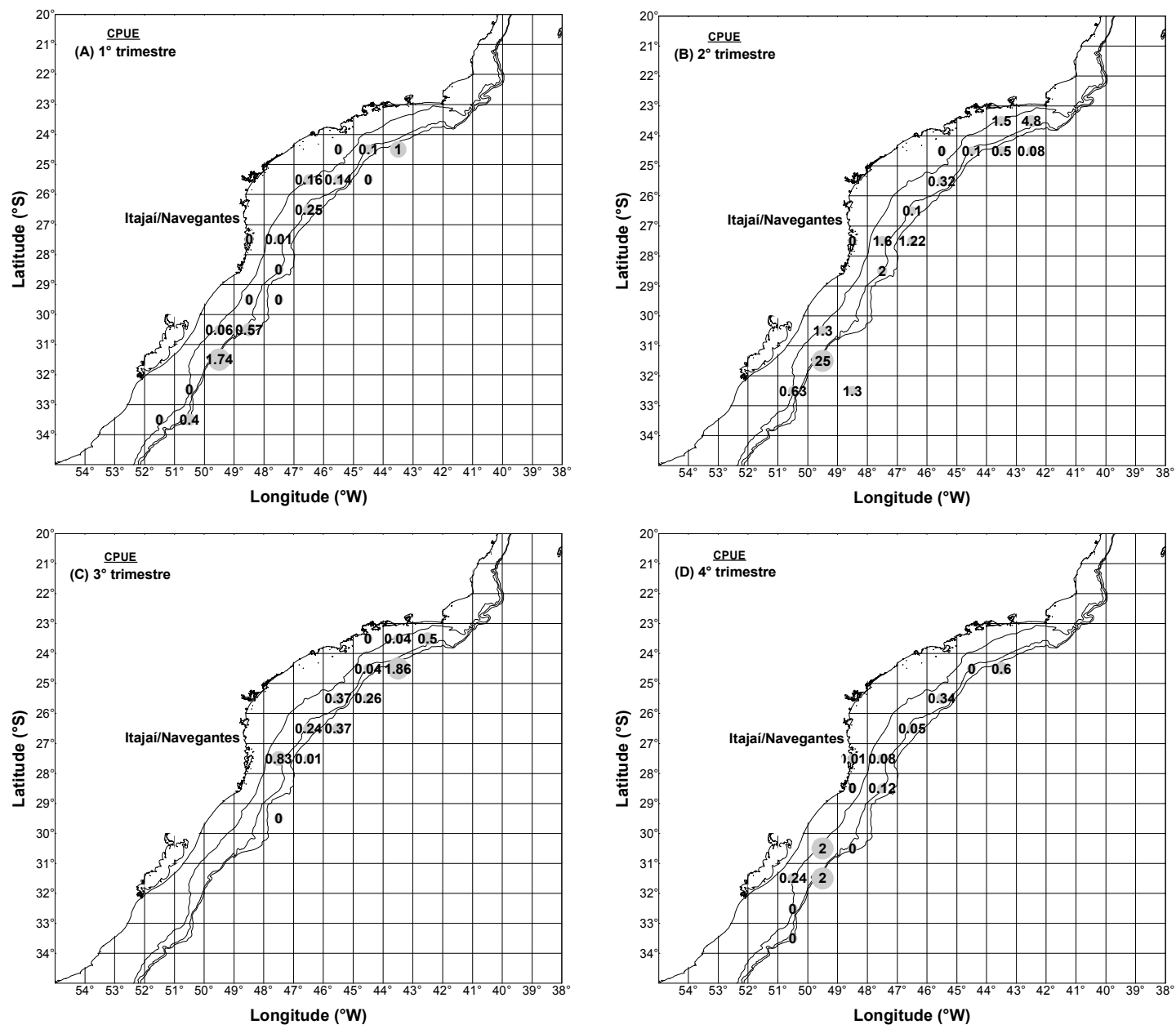

Figura 4 - Distribuição espaço-temporal das capturas por unidade de esforço (CPUE) da albacora-lage (toneladas por dia efetivo de pesca) em 2001: (A) $1^{\circ}$ trimestre, (B) $2^{\circ}$ trimestre, (C) $3^{\circ}$ trimestre e (D) $4^{\circ}$ trimestre. As linhas contínuas paralelas à linha de costa representam as isóbatas de 100, 200 e 500 metros, respectivamente. 
principalmente no segundo trimestre ao sul de $30^{\circ} \mathrm{S}$ (Figuras 4B e 7B), indicando que esta área é propícia a colocação de bóias fixas para atrair os cardumes de albacora-lage.

A estratégia de buscar áreas mais profundas no segundo trimestre de 2002 (Figura $5 B$ ) não surtiu efeito significativo sobre os rendimentos da albacora-lage, uma vez que entre $23^{\circ} \mathrm{S}$ e $27^{\circ} \mathrm{S}$ os quadrantes mais afastados da costa apresentaram CPUEs médias iguais a zero. Neste caso, apesar de os resultados obtidos neste trabalho sugerirem que a albacoralage esteja presente ao longo de toda a área de pesca (Figura 1), parece haver uma maior vulnerabilidade dos cardumes à frota de vara $\mathrm{e}$ isca-viva na borda da plataforma continental.

Assim como ocorre com o bonito-listrado (Andrade, 2003; UNIVALI/CTTMar, 2003a) a variação espaço-temporal da captura e da CPUE da albacora-lage parece ser influencia-
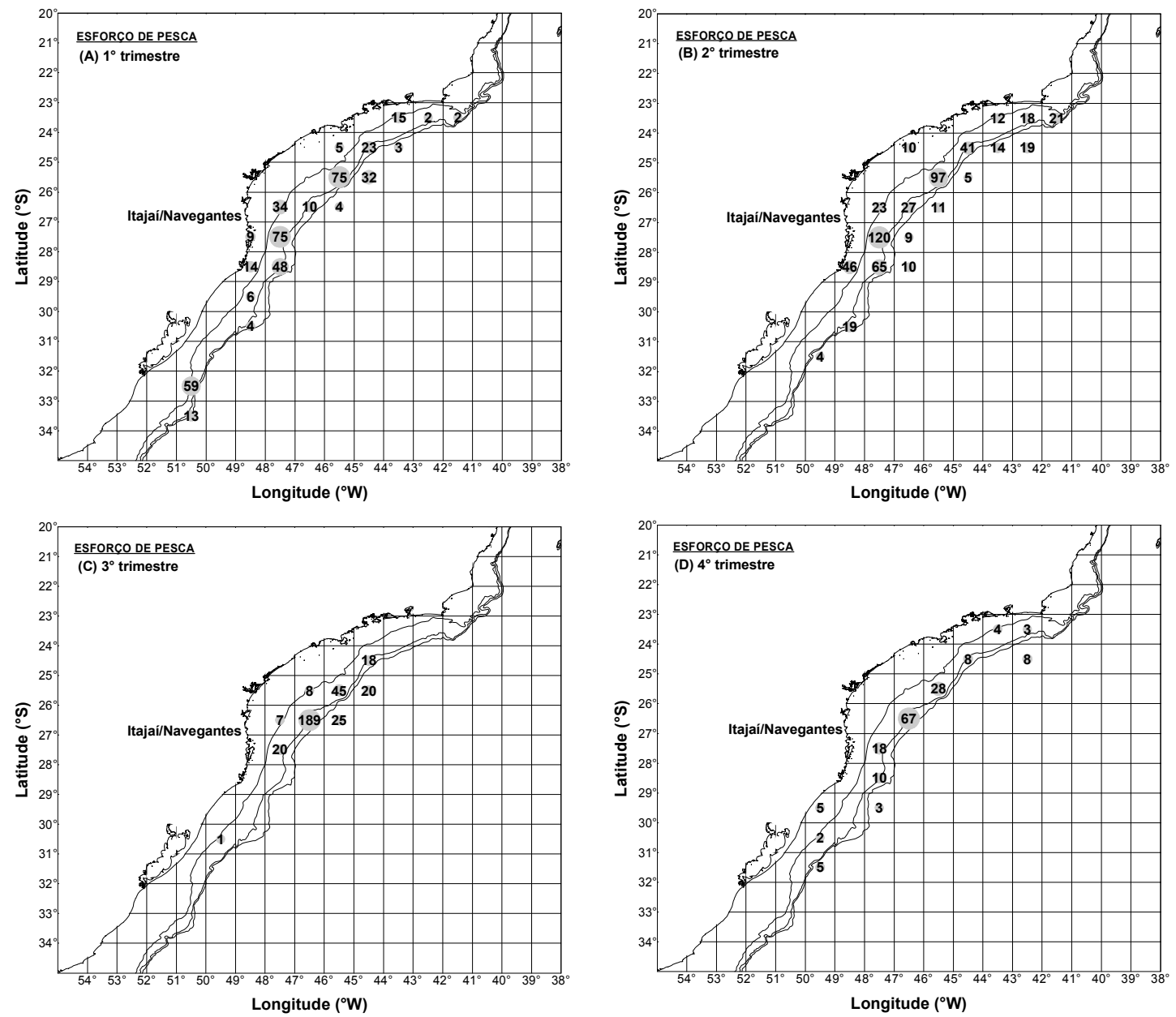

Figura 5 - Distribuição espaço-temporal do esforço de pesca da frota de vara e isca-viva (dias efetivos de pesca) em 2002: (A) $1^{\circ}$ trimestre, (B) $2^{\circ}$ trimestre, (C) $3^{\circ}$ trimestre e (D) $4^{\circ}$ trimestre. As linhas contínuas paralelas à linha de costa representam as isóbatas de 100, 200 e 500 metros, respectivamente. 
da pelos deslocamentos norte-sul da Corrente do Brasil. Neste caso, os cardumes ficariam agregados mais ao sul durante o verão, limitando-se a latitudes mais ao norte no inverno, quando a influência da Corrente do Brasil diminui na região sul. Entretanto, para reforçar esta hipótese, seria necessário analisar dados de pesca das embarcações de vara e isca-viva sediadas em Rio Grande (RS) (que continuam realizando alguns lances de pesca ao sul de $30^{\circ} \mathrm{S}$ durante o inverno) para verificar se realmente há uma menor disponibilidade do recurso na região durante o inverno, ou se os padrões apresentados refletem mais o deslocamento da frota amostrada em busca dos cardumes de bonito-listrado em áreas de pesca mais ao norte.

Em síntese, os resultados obtidos no presente trabalho indicam que o cenário mais favorável à pesca da albacora-lage pela frota
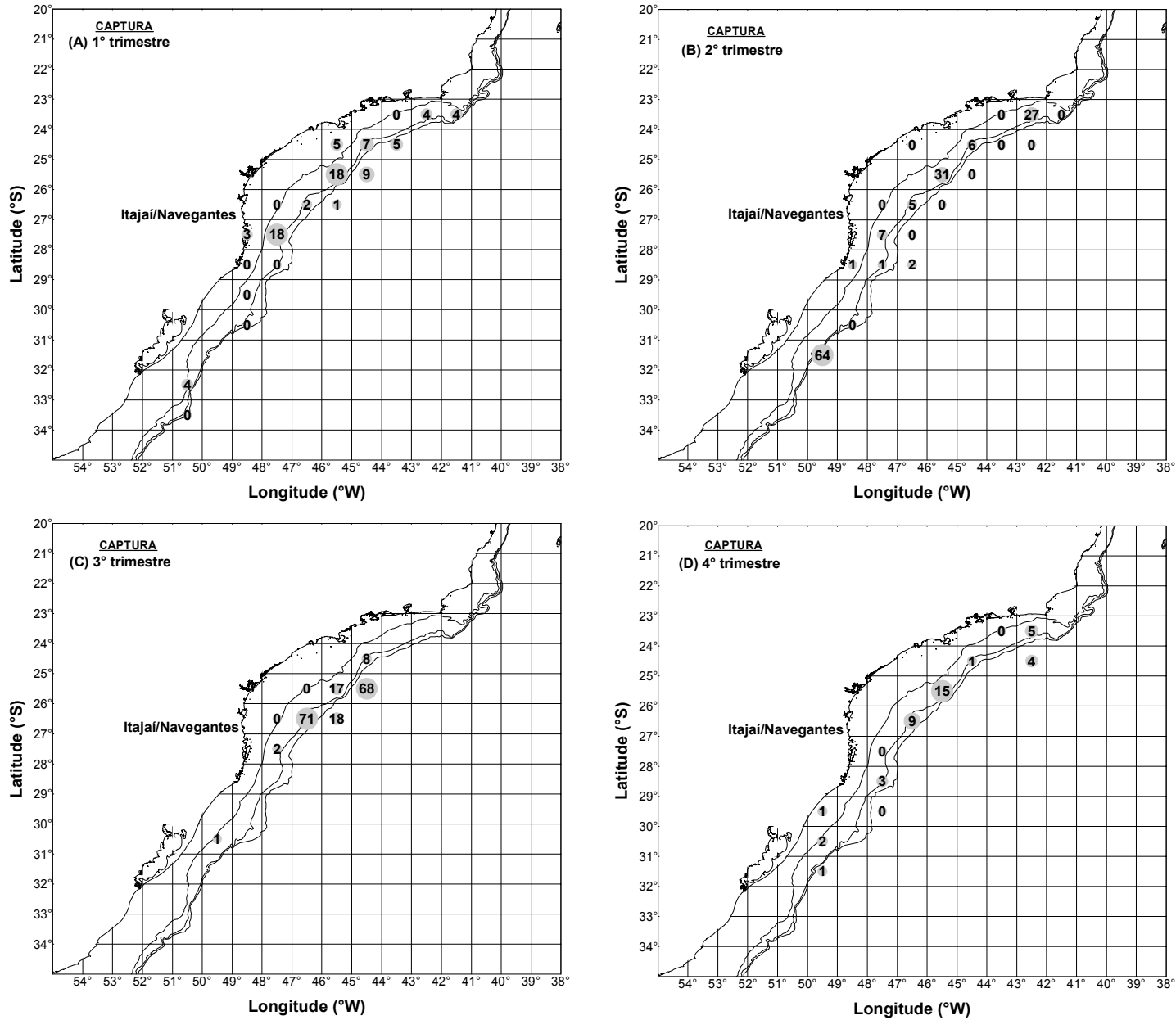

Figura 6 - Distribuição espaço-temporal das capturas de albacora-lage (toneladas) obtidas pela frota de vara e iscaviva em 2002: (A) $1^{\circ}$ trimestre, (B) $2^{\circ}$ trimestre, (C) $3^{\circ}$ trimestre e (D) $4^{\circ}$ trimestre. As linhas contínuas paralelas à linha de costa representam as isóbatas de 100, 200 e 500 metros, respectivamente. 
de vara e isca-viva no sudoeste do Atlântico é a área sul (ao sul de $30^{\circ} \mathrm{S}$ ) no segundo trimestre (Figuras 4B e 7B). Além disso, as áreas de pesca localizadas entre $23^{\circ} \mathrm{S}$ e $24^{\circ} \mathrm{S}$ - onde as capturas de albacora-lage são tradicionalmente altas (Meneses de Lima et al., 2000) tendem a ser favoráveis, principalmente no primeiro e no segundo trimestre (Figuras 4A, 4B, 7A e 7B).
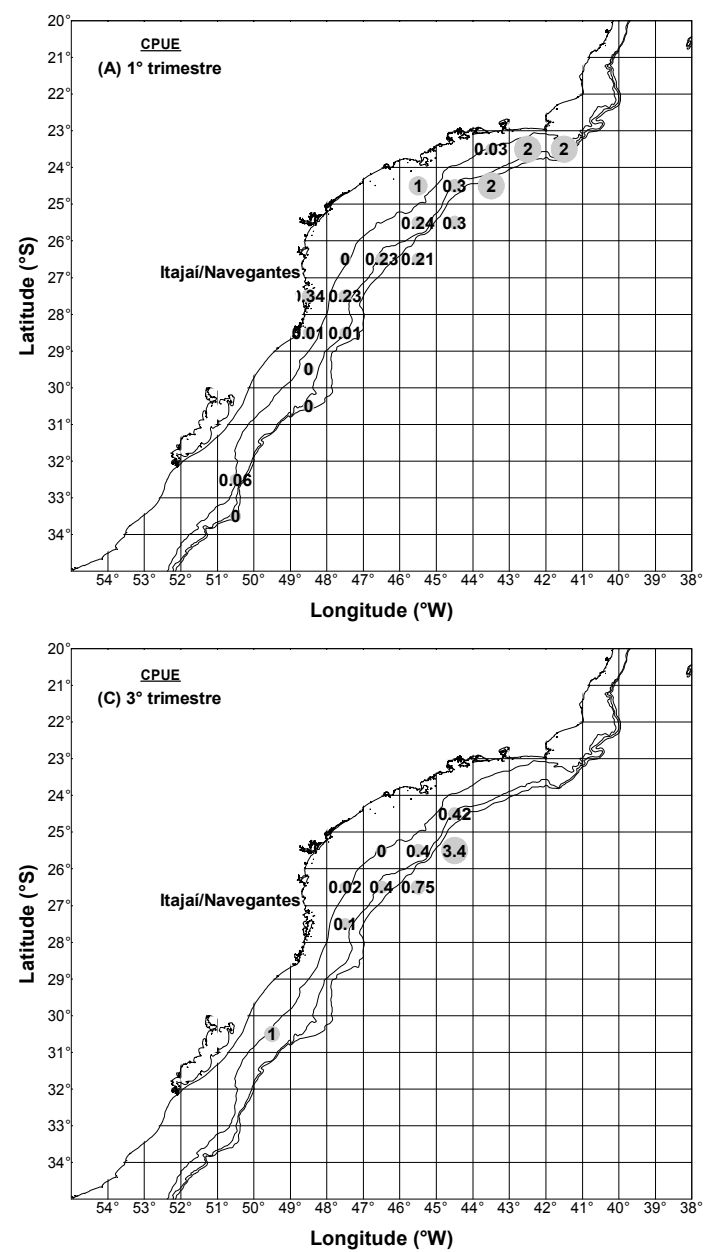

\section{AGRADECIMENTOS}

Os autores são extremamente gratos a equipe de campo do GEP/UNIVALI pela coleta dos dados de pesca utilizados neste trabalho, aos pescadores pela colaboração com nossos estudos sobre a pesca de vara e isca-viva e ao DPA/MAPA-Brasil pelo financiamento do projeto.
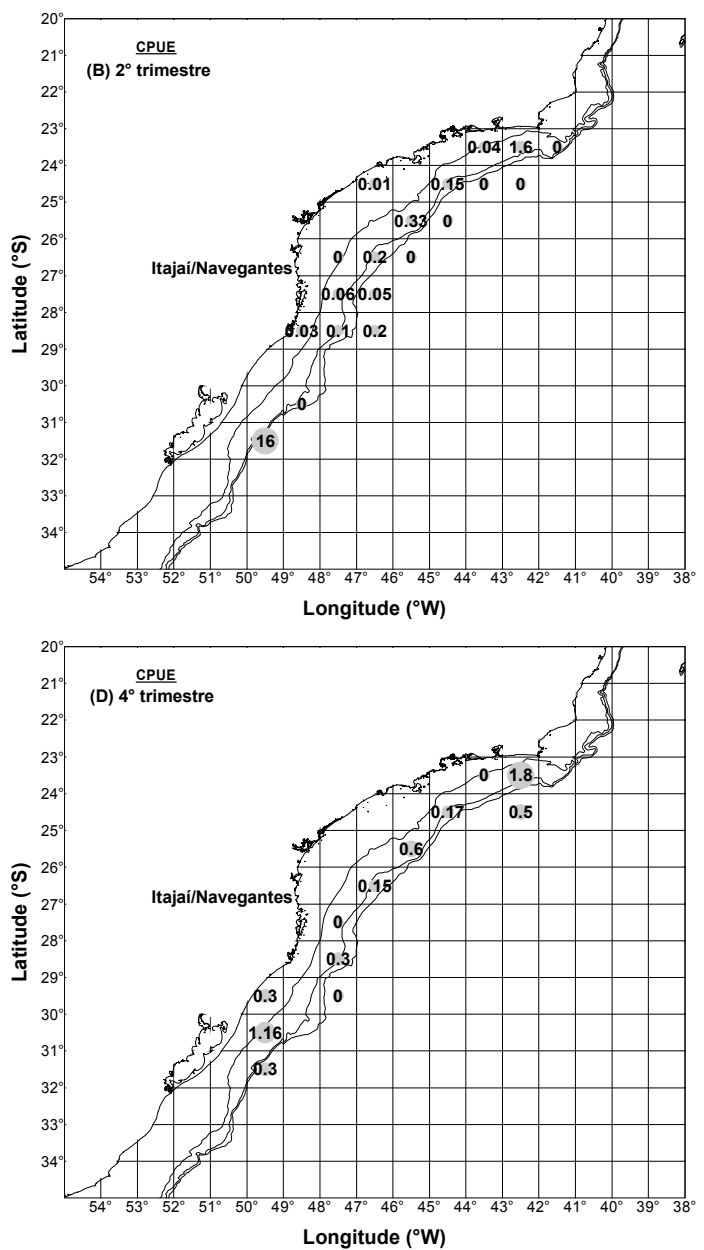

Figura 7 - Distribuição espaço-temporal das capturas por unidade de esforço (CPUE) da albacora-lage (toneladas por dia efetivo de pesca) em 2001: (A) $1^{\circ}$ trimestre, (B) $2^{\circ}$ trimestre, (C) $3^{\circ}$ trimestre e (D) $4^{\circ}$ trimestre. As linhas contínuas paralelas à linha de costa representam as isóbatas de 100, 200 e 500 metros, respectivamente. 


\section{REFERENCIAS}

Andrade, H. A. 2003. The relationship between the skipjack tuna (Katsuwonus pelamis) fishery and seasonal temperature variability in the southwestern Atlantic. Fish. Oceanogr. 12(1): 10-18.

ICCAT. 2002. 2001 detailed report on yellowfin tuna. Collec. Vol. Sci. Pap. SCRS/01/21 LI: 233-281.

Meneses de Lima, J.H.; C.F. Lin \& A.A.S. Menezes. 2000. A description of the baitboat fishery off the south and southeast Brazil. Collec. Vol. Sci. Pap. SCRS/99/68 LI: 416-463.

Paiva, M.P. 1997. Recursos pesqueiros estuarinos e marinhos do Brasil. UFC edições.

Quinn, T.J., II \& R.B. Deriso. 1999. Quantitative Fish Dynamics. Oxford University Press, New York. 542 pp.

Santos, J.A.T. \& H.A. Andrade. 2003. The variability of the species contribution to the total catch of the pole and line tuna fisheries in the Southwest Atlantic Ocean. ICCAT Coll. Vol. Sci. Pap. SCRS/2002/129 LI: 1926-1939

UNIVALI/CTTMar. 2003 a. Relatório anual da pesca brasileira do bonito-listrado
Katswonus pelamis - Ano 2002. Meta 2, Relatório Final Ações Prioritárias ao Desenvolvimento da Pesca e Aqüicultura no Sul do Brasil, Convênio Ministério da Agricultura, Pecuária e Abastecimento (MAPA), Universidade do Vale do Itajaí, MA/SARC/ 003/2000, MAPA/SARC/003/2001, MAPA/ SARC/DENACOOP/176/2002. Pezzuto, P. R. (Coordenador).

UNIVALI/CTTMar. 2003 b. Boletim estatístico da pesca industrial de Santa Catarina - Ano 2002. Ações Prioritárias ao Desenvolvimento da Pesca e Aqüicultura no Sul do Brasil, Convênio Ministério da Agricultura, Pecuária e Abastecimento (MAPA), Universidade do Vale do Itajaí, MAPA/SARC/ DENACOOP/176/2002.

Vilela, M.J.A. 1990. Idade, crescimento, alimentação e avaliação do estoque de bonito listado, Katsuwonus pelamis (Scombridae: Thunnini), explorado na região sudeste-sul do Brasil. Tese de Mestrado. Universidade do Rio Grande.

Vilela, M.J.A. \& J.P. Castello. 1991. Estudio de la edad y del crecimiento del barrilete (Katsuwonus pelamis) en la region sur y sudeste de Brasil. Frente Maritimo 9(Sec. A): 29-35. 